\title{
Detection of ctDNA in plasma of patients with clinically localised prostate cancer is associated with rapid disease progression
}

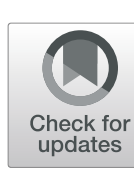

Edmund Lau ${ }^{1,2+}$, Patrick McCoy ${ }^{1 \dagger}$, Fairleigh Reeves ${ }^{1 \dagger}$, Ken Chow ${ }^{1}$, Michael Clarkson ${ }^{1}$, Edmond M. Kwan ${ }^{3,4}$, Kate Packwood ${ }^{5}$, Helen Northen ${ }^{5}$, Miao He ${ }^{5}$, Zoya Kingsbury ${ }^{5}$, Stefano Mangiola ${ }^{6}$, Michael Kerger ${ }^{7}$, Marc A. Furrer ${ }^{8,9}$, Helen Crowe ${ }^{7}$, Anthony J. Costello ${ }^{7}$, David J. McBride ${ }^{5}$, Mark T. Ross ${ }^{5}$, Bernard Pope ${ }^{1,2,3,10,}$ Christopher M. Hovens ${ }^{1}$ and Niall M. Corcoran ${ }^{1,7,8,11,12^{*}}$ (1)

\begin{abstract}
Background: DNA originating from degenerate tumour cells can be detected in the circulation in many tumour types, where it can be used as a marker of disease burden as well as to monitor treatment response. Although circulating tumour DNA (ctDNA) measurement has prognostic/predictive value in metastatic prostate cancer, its utility in localised disease is unknown.
\end{abstract}

Methods: We performed whole-genome sequencing of tumour-normal pairs in eight patients with clinically localised disease undergoing prostatectomy, identifying high confidence genomic aberrations. A bespoke DNA capture and amplification panel against the highest prevalence, highest confidence aberrations for each individual was designed and used to interrogate ctDNA isolated from plasma prospectively obtained pre- and post- ( $24 \mathrm{~h}$ and 6 weeks) surgery. In a separate cohort $(n=189)$, we identified the presence of ctDNA TP53 mutations in preoperative plasma in a retrospective cohort and determined its association with biochemical- and metastasis-free survival.

Results: Tumour variants in ctDNA were positively identified pre-treatment in two of eight patients, which in both cases remained detectable postoperatively. Patients with tumour variants in ctDNA had extremely rapid disease recurrence and progression compared to those where variants could not be detected. In terms of aberrations targeted, single nucleotide and structural variants outperformed indels and copy number aberrations. Detection of ctDNA TP53 mutations was associated with a significantly shorter metastasis-free survival (6.2 vs. 9.5 years (HR 2.4; 95\% Cls 1.2-4.8, $p=0.014)$.

Conclusions: CtDNA is uncommonly detected in localised prostate cancer, but its presence portends more rapidly progressive disease.

* Correspondence: niallmcorcoran@gmail.com

${ }^{\dagger}$ Edmund Lau, Patrick McCoy and Fairleigh Reeves contributed equally to this

work.

${ }^{1}$ Department of Surgery, University of Melbourne, 5th Floor Clinical Sciences

Building, Royal Melbourne Hospital, Grattan Street, Parkville, VIC 3050,

Australia

${ }^{7}$ Australian Prostate Cancer Centre, North Melbourne, VIC 3195, Australia

Full list of author information is available at the end of the article

(c) The Author(s). 2020 Open Access This article is licensed under a Creative Commons Attribution 4.0 International License, which permits use, sharing, adaptation, distribution and reproduction in any medium or format, as long as you give appropriate credit to the original author(s) and the source, provide a link to the Creative Commons licence, and indicate if changes were made. The images or other third party material in this article are included in the article's Creative Commons licence, unless indicated otherwise in a credit line to the material. If material is not included in the article's Creative Commons licence and your intended use is not permitted by statutory regulation or exceeds the permitted use, you will need to obtain permission directly from the copyright holder. To view a copy of this licence, visit http://creativecommons.org/licenses/by/4.0/ The Creative Commons Public Domain Dedication waiver (http://creativecommons.org/publicdomain/zero/1.0/) applies to the data made available in this article, unless otherwise stated in a credit line to the data. 


\section{Background}

The majority of men diagnosed with localised prostate cancer are effectively cured with surgery and/or radiation therapy [1]; however, a proportion of patients will experience disease recurrence, often associated with high grade and/or locally advanced tumours [2]. Predicting patients at highest risk of relapse remains challenging, as while clinical prognostic factors are certainly helpful [3], they do not always provide the full picture for an individual patient. The ability to accurately identify these patients may have clinical implications, as they have the potential to benefit from systemic treatment intensification strategies. While the value of these systemic treatments in metastatic hormone-sensitive prostate cancer (mHSPC) is undisputed (ENZAMET [4], ARCHES [5], TITAN [6], STAMPEDE [7, 8], LATITUDE [9], CHAA RTED [10]), utility in early-stage disease is less clear. However, this is an area of intense investigation in which treatment strategies will continue to evolve [11, 12]. Prognostic biomarkers to predict risk of metastatic disease development are thus urgently required, not only to facilitate discussions with patients/caregivers around expected outcomes, but also to inform clinical trial design and perhaps ultimately guide optimal management.

A number of molecular biomarkers for use on primary prostate cancer tissue are already in clinical use (for example Decipher [13], Oncotype [14], and Prolaris [15]) and can be used to predict the presence of adverse pathology or recurrent disease post primary treatment. These transcription-based assays require the use of tumour tissue from the diagnostic biopsy specimen, which in the context of the well-described molecular heterogeneity of primary prostate cancer [16] as well as the sampling error inherent with traditional biopsy strategies [17], has led to the exploration of circulating biomarkers as a 'whole tumour' sampling technique. An array of different biomarkers have been investigated, including circulating tumour cells (CTCs), exosomes and circulating tumour DNA (ctDNA), although most of these have been in the metastatic castration-resistant setting (mCRPC), where tumour burden is higher [18, 19]. Use of these biomarkers in the localised setting, where disease volume is considerably lower, may pose significant challenges. In this context, the use of ctDNA has some appeal, due to the ability to amplify the signal against background noise, as well as the increasing annotation of particular genomic features as important drivers of clinical outcome [20]. However, the current analytical sensitivity and limit of detection of most 'off the shelf ctDNA assays is inadequate to reliably call low-frequency variants observed in early prostate cancer [21]. One of the ways to overcome this is to use ultradeep sequencing using targeted panels looking for patient-specific alleles.
This personalised assay approach has recently been investigated in localised prostate cancer [22]. As part of a broader attempt to identify ctDNA in a large cohort of patients with localised disease, Hennigan and colleagues performed multiregional sequencing to identify patientspecific variants present clonally or at high allele frequency within the primaries of nine patients, followed by targeted deep sequencing of these variant sites within plasma-derived cell-free DNA. Despite readily detecting ctDNA in patients with metastatic disease, they were unable to positively identify ctDNA in any patient by this or any other approach. Although the authors provide a number of plausible biological reasons for this negative finding, including low rates of cell proliferation and ctDNA shedding in localised disease, as well as more restricted access to the vasculature compared to metastatic lesions, a further possibility is that the number of patients with truly high-risk disease within their cohort was too low to detect a positive signal. Certainly ctDNA measurement has proven feasible and clinically meaningful in detecting the presence of 'micrometastatic disease', and be prognostic for recurrence, in other tumour types in the localised setting [23, 24].

In this study, we performed panel-based targeted sequencing informed by individual patient tumour WGS, as well as tagged-amplicon deep sequencing (TAm-Seq) across the TP53 gene in two separate cohorts of patients with localised prostate cancer, with an emphasis on high-risk disease [25]. We found that ctDNA was detectable in both the pre- and post-operative setting, and that pre-operative detection was associated with a significant reduction in metastasis-free survival.

\section{Methods}

\section{Study cohorts}

Two separate patient cohorts were used for this study. For tumour-based WGS analysis (WGS cohort), we prospectively enrolled 10 consecutive patients presenting to a single surgeon with clinically localised prostate cancer suitable for prostatectomy with curative intent. For the TAm-Seq analysis (TAm-Seq cohort, $n=189$ ), patients were selected from a prospectively collected and clinically annotated institutional bio-repository [26]. Patients were prioritised based on the length of follow-up, enriched for high-risk disease and recurrence events. Collection and use of material had Institutional Review Board approval (HREC\# 626-14).

\section{Whole-genome sequencing and analysis}

A summary of the blood and prostatectomy specimens available for WGS is shown in Additional file 1: Table S1. Patients with both tumour and matched germline DNA samples from a peripheral blood buffy coat specimen were suitable for WGS to discover patient-specific 
variants. Genomic DNA extracted from blood and tissue samples were prepared using the $\mathrm{TruSeq}^{\circ}$ DNA PCRfree sample preparation kit (Illumina, San Diego, CA, USA). The libraries were sequenced as paired-ends $(2 \times$ 150 cycles) using a HiSeqX platform to an average depth of $>30 \mathrm{x}$ for germline and $>100 \mathrm{x}$ for tumour samples, following alignment to the Human Reference sequence (hg19) using iSAAC-03.16.02.19 and removal of duplicate read-pairs. Variant calling was performed using the Illumina IsaacVariantCaller (2.6.53.23) [27].

\section{Enrichment panel design and targeted sequencing and analysis}

A subset of somatic variants identified through wholegenome sequencing were included on the panel for each patient. Variants were preferentially selected for inclusion where they occurred in genes previously implicated in cancer [28] or prostate cancer specifically [21]. A range of SNVs, indels and structural variants (SVs) were targeted. The final design targeted $88 \mathrm{~kb}$ of the genome. Target regions were uploaded to Illumina DesignStudio (www.designstudio.illumina.com) for probe design. A single probe was selected for small variants, while SVs were targeted using a probe from each side of the rearrangement junction. For indels, a probe adjacent to the variant was selected (Additional file 2: Figure S1). Probes were validated on fresh frozen tumour DNA to confirm successful design and synthesis, and a list of genomic variants targeted in each patient is provided in Additional file 3: Table S2.

For each patient, blood was collected from up to three timepoints in EDTA tubes for ctDNA analysis; sample (a) was taken pre-surgery, sample (b) $24 \mathrm{~h}$ post-surgery and sample (c) 6 weeks post-surgery. Immediately after collection, plasma was collected following a double spin ( $820 \mathrm{~g}$ for $10 \mathrm{~min}$; $16,000 \mathrm{~g}$ for $10 \mathrm{~min}$ ) and stored at $80^{\circ} \mathrm{C}$ until analysis. Circulating DNA was extracted using the QIAamp Circulating Nucleic Acid Kit (Qiagen, Hilden, Germany), and DNA quality was assessed using an Agilent BioAnalyzer and quantified using the Qubit method (Thermofisher, Delaware, USA).

Input into library preparations was between 28 and $60 \mathrm{ng}$ of ctDNA, with a median value of $39 \mathrm{ng}$ as determined by Qubit. Enriched libraries were prepared from ctDNA extracted from plasma using the TruSight ${ }^{\circ}$ Tumor 170 library prep kit supplemented with the TruSight ${ }^{\circ}$ Oncology UMI Reagents (Illumina Inc.). Adapters containing UMIs enable detection of genuine, very low-frequency variants through the reduction of background noise in sequencing data. Due to the small fragment length of the starting material, the DNA shearing step of the TST170 protocol was omitted. Libraries were enriched with the custom panel then sequenced as paired-ends $(2 \times 150 \mathrm{bp})$ plus indexing reads on NextSeq High output. Five or six samples were loaded per flow cell to achieve $\sim 40$, 000x raw depth per sample.

Samples were processed using an Illumina R\&D analysis pipeline to take advantage of the UMIs incorporated during library preparation. Reads were grouped into read families by alignment to the hg19 human reference sequence followed by collapsing and stitching into unique fragments utilising start and stop positions, UMI barcodes and the paired read (where present) to generate collapsed BAM files. These collapsed families of reads are low noise and high confidence. Positions targeted by the panel were reviewed using Integrative Genomics Viewer (IGV; The Broad Institute) to confirm the presence or absence of the targeted variants.

\section{TP53 TAm-Seq}

DNA was isolated from archived plasma samples stored in liquid nitrogen using the QIAamp Circulating Nucleic Acid Kit and quantified using Qubit Molecular Probes dsDNA high sensitivity assay kit (Invitrogen, CA, USA). For amplicon production, forward and reverse primers (Additional file 2: Figure S2) tiling across the TP53 gene were combined for use in eight separate PCR reactions per patient. Input was $2 \mathrm{ng}$ of DNA, which was added to a PCR reaction mix composed of $2 \mu \mathrm{l}$ of primer $(0.5 \mu \mathrm{l}$ ea. @ $10 \mu \mathrm{M})$ and $10 \mu \mathrm{l}$ Q5 DNA pol 2X master mix made up to $20 \mu \mathrm{l}$ with MQ water. The reaction was run on a ViiA 7 PCR machine with the following settings: initial denaturation at $95^{\circ} \mathrm{C} \times 1 \mathrm{~min}$, then cycle denaturation at $95^{\circ} \mathrm{C} \times 10 \mathrm{~s}$ following by annealing at $58^{\circ} \mathrm{C} \times 1 \mathrm{~s}$ and then extension at $72{ }^{\circ} \mathrm{C} \times 18 \mathrm{~s}$ for 38 cycles. Amplicons were then combined into two pools of non-overlapping products and cleaned with the Qiagen QIAquick PCR purification kit following the manufacturer's instructions. DNA was quantified using a nanodrop and $100 \mathrm{ng}$ of DNA of each pool end repaired and adaptor ligated using NEBNext Adaptors for Illumina according to the manufacturer's instructions. Following a AMPure XP bead clean-up, a second round of PCR amplification was performed in a total reaction volume of $50 \mu \mathrm{l}$ consisting of $20 \mu \mathrm{l}$ of adaptor ligated DNA fragments, $25 \mu \mathrm{l}$ Q5 $2 \mathrm{X}$ master mix, $2.5 \mu \mathrm{l}$ index primer $(10 \mu \mathrm{M})$ and $2.5 \mu \mathrm{l}$ universal PCR primer $(10 \mu \mathrm{M})$. PCR amplification was then performed in a thermocycler under the following conditions: initial denaturation at $98^{\circ} \mathrm{C} \times 30 \mathrm{~s}$, then cycle denaturation at $98^{\circ} \mathrm{C} \times 10 \mathrm{~s}$ following by annealing/extension at $65^{\circ} \mathrm{C} \times 75 \mathrm{~s}$ for 8 cycles, with a final extension at $65^{\circ} \mathrm{C}$ for $5 \mathrm{~min}$. The reaction mixture was then cleaned again using AMPure XP beads following the manufacturer's instructions at a ratio of 0.9:1 beads to ligation mixture. The pool was then 
quantified and quality checked on a Bioanalyzer (Agilent, CA, USA) before sequencing on a MiSeq (Illumina Inc.). A detailed description of the experimental procedure is provided as supplementary information (Additional file 4; Supplementary Methods).

\section{TAm-Seq analysis}

For each sample in the study, paired-end DNA sequencing reads (length $151 \mathrm{bp}$ ) were aligned to the human genome reference hg19 using BWA mem (version 0.7.17). Reads were assigned to their corresponding PCR amplicons based on their alignment coordinates. To be considered for further analysis, reads were required to have a minimum alignment length of $75 \mathrm{bp}$, a minimum overlap of at least $75 \%$ of their target amplicon and no more than $2 \mathrm{bp}$ edit distance from the reference genome. Using the filtered set of reads, the frequency of each DNA base was counted at each genomic locus within the target PCR amplicon regions for each sample. Using the control samples, at each locus, a null model was computed to represent the expected distribution of allele counts when no variant is present. Allele count data was modelled (with add-one smoothing) using the Dirichlet Multinomial distribution with best hyperparameter computed by maximising the log-likelihood function using the Newton-Raphson method. For each locus in the case samples, the allele count was compared with the null model. The weight of evidence against the null was measured by the Bayes factor B. The prior distribution of the count data of the case samples was given by a Dirichlet distribution on a 3-simplex, with all pseudocounts set to $1 / 2$ (as per Jeffrey's Prior on a multinomial distribution). Variant allele frequencies (VAF) were computed using the maximum a posteriori (MAP) estimate on the posterior distribution marginalised to the component representing the non-reference allele. After filtering out any variants with gnomAD (gnomad.broadinstitute.org) population frequency greater than $1 \%$, a set of high-confidence variants was selected based on having a minimum filtered depth of coverage of 500 reads, a minimum log Bayes factor of 6 (equivalent to posterior odds against the null hypothesis of 50:1) and a cohort frequency of less than four samples. The predicted pathogenicity of detected variants was assessed using Varsome [29].

\section{Clinical outcomes}

Postoperative PSA data was collected prospectively in all TAm-Seq patients. Biochemical recurrence was defined as a single post-operative PSA reading of $\geq 0.2 \mathrm{ng} / \mathrm{ml}$, or a rising measurement below this that was determined to represent a recurrence by the treating physician and led to the institution of salvage therapy. The presence of metastasis and mode of diagnosis was determined by retrospective chart review. For patients without recurrence or metastasis, follow-up was censored at the time of their last PSA or clinical review as appropriate. To compare biochemical recurrence-free and metastasisfree survival between groups, Kaplan-Meier curves were generated and compared using a two-sided logrank test, with significance assumed at $p<0.05$.

\section{Results}

Paired tumour and germline WGS was completed on eight patients, the clinical and pathological characteristics of whom are shown in Table 1. Consistent with contemporary practice, all patients had intermediate- or high-risk disease at the time of radical treatment, and two patients underwent salvage prostatectomy for radiorecurrent disease. Patients at risk of metastasis based on their disease characteristics were staged pre-operatively with a technetium-99m-MDP whole body bone scan and cross-sectional imaging (MRI pelvis \pm lumbar spine or CT abdomen/pelvis) as appropriate. No patient had evidence of metastatic disease at the time of surgery.

The number of genomic aberrations identified by tumour WGS per patient is summarised in Additional file 1: Table S3. The average mutation rate was 5.51 SNVs per megabase, consistent with a high-risk disease cohort [21]. The category of features selected for targeted capture and sequencing and a breakdown of variants targeted versus those detected in ctDNA are summarised in Fig. 1. Genomic features of prostate cancer were identified in ctDNA in two of eight patients investigated with only SNVs and SVs being detected. Interestingly, while all SNVs and SVs targeted in patient 11196_3 were detectable in the ctDNA, we could find no evidence of the targeted small indels, despite their presence at high frequency in the tumour WGS data and good coverage in these regions. Similarly CNAs detected in ctDNA in general correlated poorly with tumour WGS and could not be reliably called at the ctDNA levels observed in this localised cohort.

Changes in specific variants identified in the ctDNA in patients 11196_3 and 11201_4 over time are shown in Figs. 2 and 3, along with a summary of the clinical course of their disease post-surgery. Two timepoints (pre-operative and $24 \mathrm{~h}$ post-surgery) were available for patient 11196_3, who showed some evidence of a treatment effect, with a decrease in the variant allele frequency observed for almost all of the ctDNA variants measured (Fig. 2a). A similar effect was not consistently observed in patient 11201_4, with an inexorable increase in variant allele frequency of measured variants observed across the three timepoints. Although all patients were negative for metastatic disease using conventional imaging at the time of surgery, patients in whom ctDNA was detectable pre-treatment had strikingly aggressive 
Table 1 Clinical and pathological characteristics of the WGS study cohort

\begin{tabular}{|c|c|c|c|c|c|c|c|c|c|}
\hline Sample & Age & PSA & Prior RT & CT & $B \times G G$ & Staging & RP GG & pT & $\mathrm{Vol}(\mathrm{cc})$ \\
\hline 11193_2 & 70 & 26.9 & $y$ & CT1C & 5 & $\begin{array}{l}\text { MRI } \\
\text { WBBS }\end{array}$ & 5 & pT3b & 21.3 \\
\hline 11196_3 & 57 & 9.3 & y & cT1c & 3 & $\begin{array}{l}\text { MRI } \\
\text { WBBS }\end{array}$ & 3 & pT3b & 2.1 \\
\hline 11201_4 & 64 & 8.1 & $n$ & сT3a & 5 & $\begin{array}{l}C T \\
\text { WBBS }\end{array}$ & 5 & pT3b & 18.5 \\
\hline 11199_5 & 67 & 7.8 & $n$ & cT2a & 2 & MRI & 3 & рT3a & 15.2 \\
\hline 11219_6 & 58 & 6.5 & $n$ & cT1c & 2 & MRI & 2 & рT3a & 2.1 \\
\hline 11231_9 & 59 & 6.1 & $\mathrm{n}$ & CT1C & 2 & MRI & 2 & pT2c & 3.7 \\
\hline 11242_10 & 61 & 4.1 & $n$ & cT2a & 2 & $\begin{array}{l}\mathrm{CT} \\
\text { WBBS }\end{array}$ & 2 & рT3a & 3.7 \\
\hline 11243_11 & 76 & 7.9 & $\mathrm{n}$ & cT2a & 5 & $\begin{array}{l}C T \\
\text { WBBS }\end{array}$ & 3 & pT3a & 7.5 \\
\hline
\end{tabular}

PSA prostate-specific antigen, $R T$ radiotherapy, $C T$ clinical stage, Bx diagnostic biopsy, GG International Society of Urological Pathology Grade Group, RP radical prostatectomy, $p T$ pathological stage, $\mathrm{Vol}(\mathrm{cc})$ tumour volume $\left(\mathrm{cm}^{3}\right)$

post-treatment clinical courses compared to patients with no detectable ctDNA (Additional file 2: Figure S3), characterised by early disease recurrence and metastases, relatively poor response to systemic treatments, and progression to death within 2.5 years.

To extend these findings, we performed targeted sequencing of plasma DNA collected from patients undergoing prostatectomy for presumed localised prostate cancer $(n=189)$, focussing on TP53, one of the most commonly mutated genes in prostate cancer, and one that is positively enriched for in metastatic disease (including in patient 11196_3) [20,30]. As indicated in the study description above, the cohort was selected to enrich for patients with high-risk and recurrent disease, to maximise the chances of detecting ctDNA in some patients, the clinical and pathological characteristics of whom are summarised in Table 2. After filtering, the mean depth of coverage within the target regions was 3198 reads. Positive detection of TP53 ctDNA variants was identified in 22 cases (12\%), with no variants detected in the remainder (Additional file 1: Table S4). The median and mean coverage depth for called variants was $9892 x$ and 12,695x respectively. Fourteen out of 21 variants identified were predicted to be pathogenic or

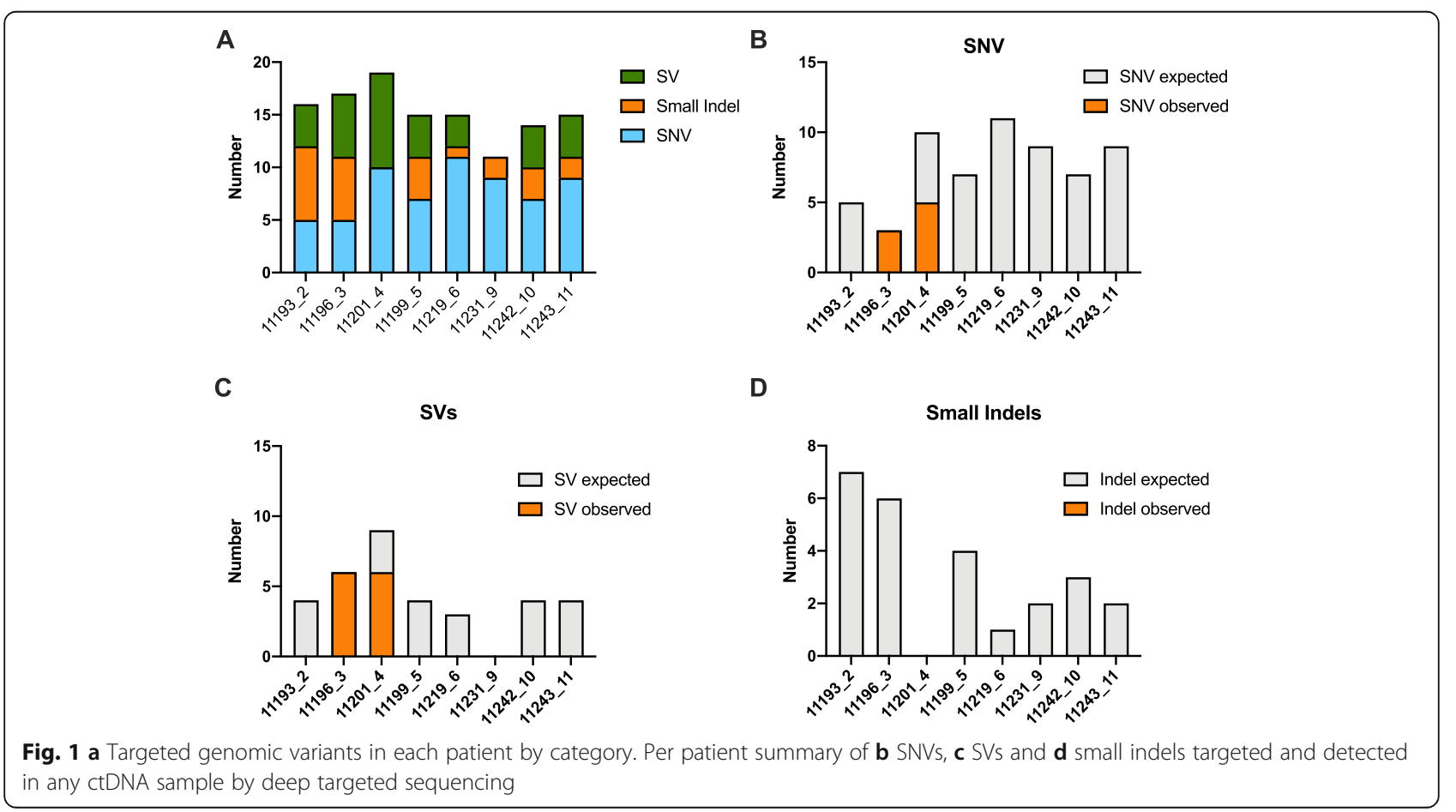


A Variant Frequency in WGS

Variant Frequency in ctDNA
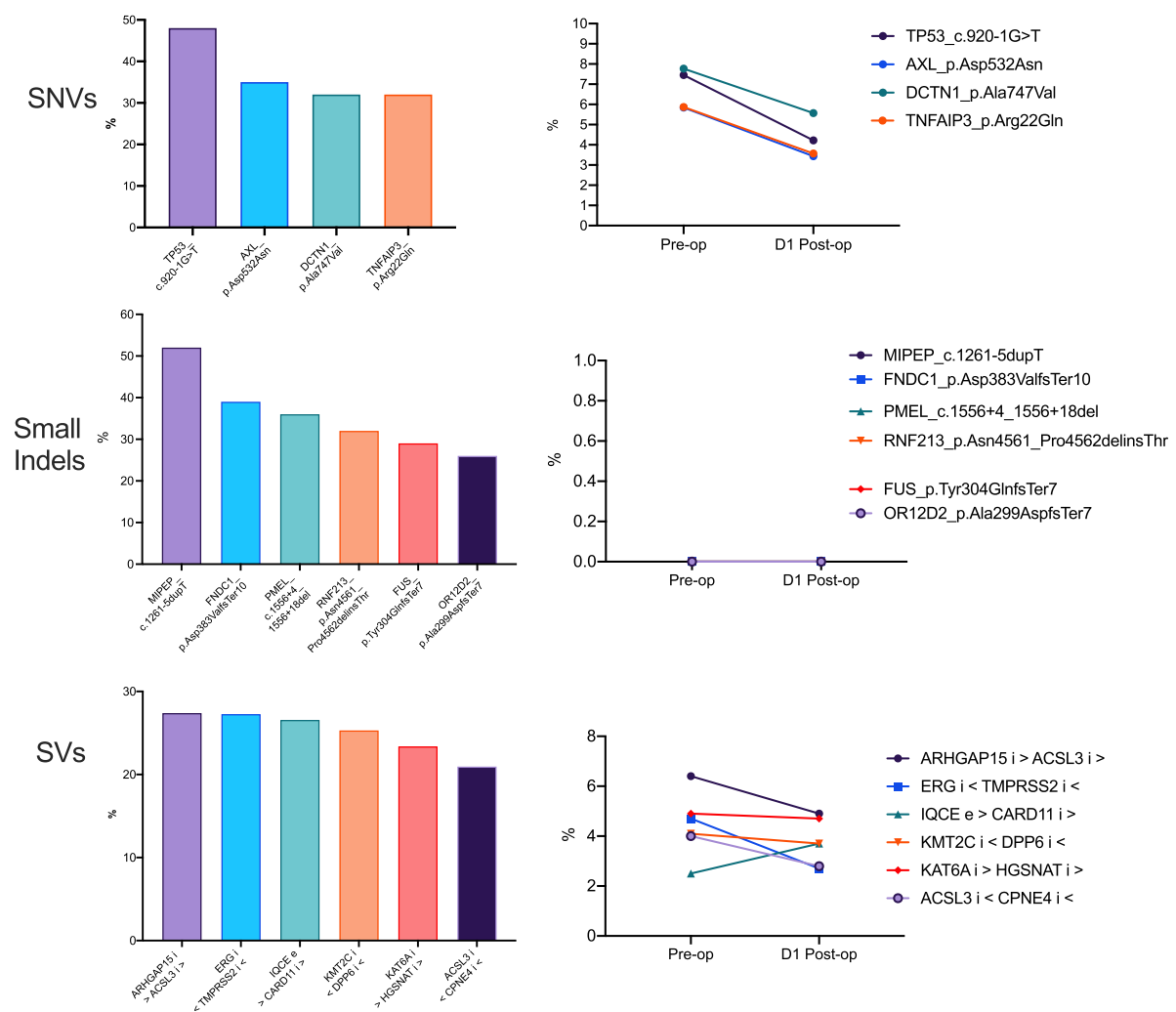

B

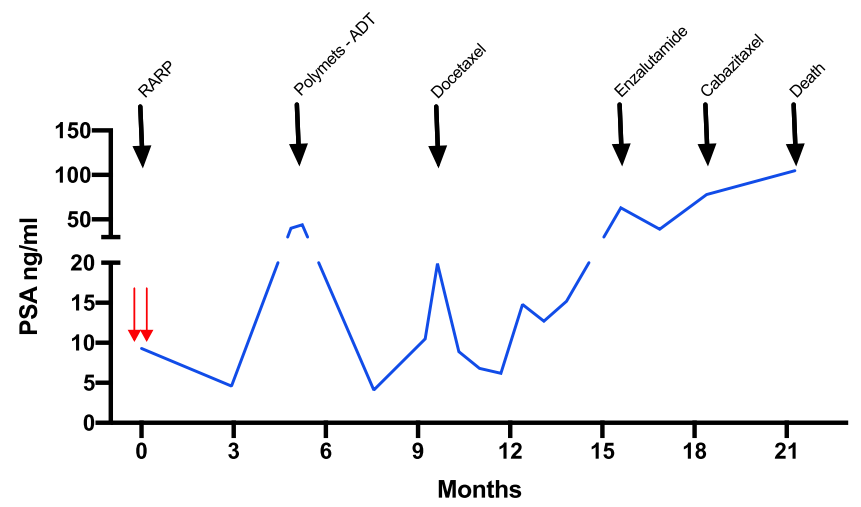

Fig. 2 a Selected variant frequency observed in tumour whole-genome sequencing (WGS) and circulating tumour DNA (ctDNA) at indicated timepoints in patient 3. b Summary of clinical history of patient 3 post-surgery with ctDNA sampling indicated by red arrows

likely pathogenic, with only one variant classed as likely benign. There was no significant difference in biochemical recurrence-free survival (BFS) between patients with or without a detectable variant (Fig. 4a), with a mean BFS of 2.4 vs. 3.7 years (HR 1.4; 95\% CIs 0.76-2.6, $p=$ 0.28 ). In contrast, metastasis-free survival (MFS) was significantly shorter in patients positive for a variant (Fig. 4b), with mean MFS of 6.2 vs. 9.5 years (HR 2.4; 95\% CIs 1.2-4.8, $p=0.014)$. Similar results were observed using a contingency analysis (Additional file 1: Table S5).

\section{Discussion}

Although ctDNA is reported to be relatively abundant in metastatic prostate cancer, at the time of initiation of this study, it was unclear if ctDNA could be detected in patients with localised disease, as well as the clinical implications of its presence. A number of early studies 


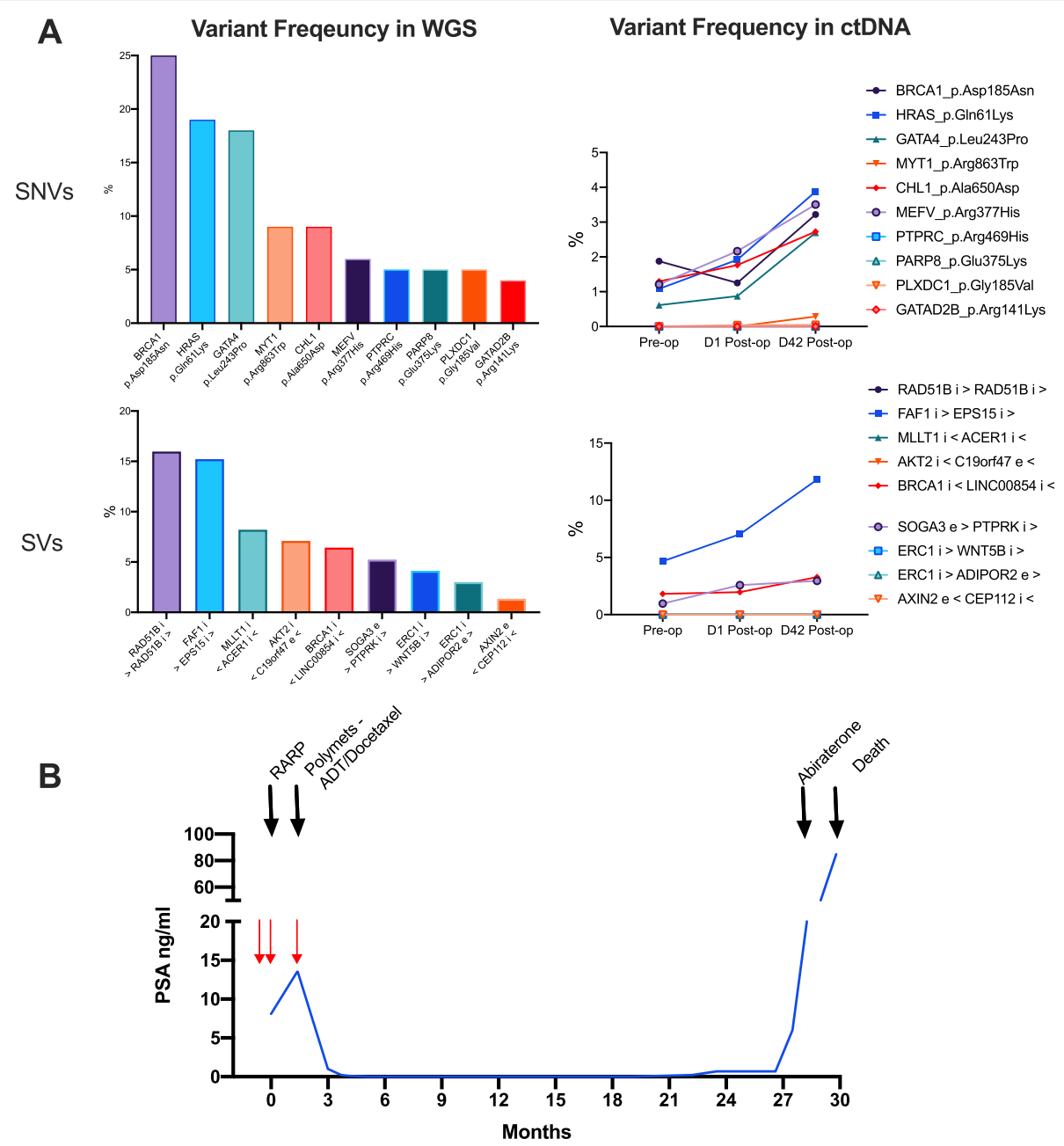

Fig. 3 a Selected variant frequency observed in tumour whole-genome sequencing (WGS) and circulating tumour DNA (ctDNA) at indicated timepoints in patient 4. b Summary of clinical history of patient 4 post-surgery with ctDNA sampling indicated by red arrows

identified an association between total cell-free plasma DNA concentration and the presence of cancer and biochemical recurrence post prostatectomy [31-33]; however, the first study to investigate circulating tumour DNA in this setting has only recently been reported [22]. In this study, Hennigan and colleagues used ultralow pass whole-genome sequencing of cell-free plasma DNA in over 100 patients with clinically localised disease prior to prostatectomy, as well as deep targeted sequencing of a bespoke panel of single nucleotide variants/indels selected as clonal or major subclonal events, based on individual multiregional whole exome sequencing in a subset of nine patients. Despite the technical robustness of the methodology evidenced by consistent identification of ctDNA in patients with established metastatic disease using these approaches, they were unable to identify any ctDNA in any patient with clinically localised disease.
Here we have been able to identify ctDNA in two of eight patients with localised disease using a personalised approach based on individual whole-genome sequencing followed by deep-targeted sequencing of selected variants in plasma cell-free DNA. Furthermore, in a retrospective analysis of patient samples (enriched for highrisk patients) using the previously described TAm-Seq method to tile across a single gene, we have been able to detect ctDNA in 22 out of a further 189 patients. Importantly, we were able to demonstrate that detection of ctDNA in the pre-prostatectomy setting has prognostic implications and is associated with more rapid progression to metastatic disease in both analyses. This is of clear clinical relevance, given that MFS is a strong surrogate of overall survival in localised prostate cancer [34].

Despite differences in sequencing and analysis methods between the studies, at least for the individual targeted analysis, it is likely that clinical differences 
Table 2 Clinical and pathological characteristics of the TAm-Seq study cohort

\begin{tabular}{|c|c|}
\hline$n$ & 189 \\
\hline \multicolumn{2}{|l|}{ Age (years) } \\
\hline Median & 63 \\
\hline IQR & $58-67.6$ \\
\hline \multicolumn{2}{|l|}{ PSA ng/ml } \\
\hline Median & 7.5 \\
\hline IQR & $5.3-12.95$ \\
\hline \multicolumn{2}{|l|}{ cT } \\
\hline 1 & $105(55.6 \%)$ \\
\hline 2 & $63(33.3 \%)$ \\
\hline 3 & $21(11.1 \%)$ \\
\hline \multicolumn{2}{|c|}{ Biopsy ISUP Grade Group } \\
\hline 1 & $33(17.5 \%)$ \\
\hline 2 & $61(32.3 \%)$ \\
\hline 3 & $29(15.3 \%)$ \\
\hline 4 & $35(18.5 \%)$ \\
\hline 5 & $31(16.4 \%)$ \\
\hline \multicolumn{2}{|l|}{ pT } \\
\hline 2 & $88(46.6 \%)$ \\
\hline $3 a$ & $60(31.7 \%)$ \\
\hline $3 b$ & $41(21.7 \%)$ \\
\hline \multicolumn{2}{|c|}{ Prostatectomy ISUP Grade Group } \\
\hline 1 & $10(5.3 \%)$ \\
\hline 2 & $63(33.3 \%)$ \\
\hline 3 & $61(32.3 \%)$ \\
\hline 4 & $11(5.8 \%)$ \\
\hline 5 & $44(23.3 \%)$ \\
\hline \multicolumn{2}{|c|}{ Tumour volume (cc) } \\
\hline Median & 3.35 \\
\hline IQR & $1.5-7.37$ \\
\hline \multicolumn{2}{|c|}{ Biochemical recurrence } \\
\hline No & $90(47.6 \%)$ \\
\hline Yes & 99 (52.4\%) \\
\hline \multicolumn{2}{|l|}{ Metastases } \\
\hline No & $117(61.9 \%)$ \\
\hline Yes & 49 (35.9\%) \\
\hline Not reported & $23(12.2 \%)$ \\
\hline
\end{tabular}

between the cohorts are the basis of the observed discrepancy. For instance in the Hennigan study, although 8/9 patients who underwent tissue and plasma cell-free DNA sequencing had locally advanced and/or high grade tumours, only one patient experienced a biochemical recurrence with 24 months of surgery, and no patients were reported to progress to metastasis. In contrast, from our cohort, three patients had primary PSA persistence, and a further two patients experienced biochemical recurrence within 24 months. Of these, ctDNA was only detectable in 2 patients, both of whom had primary PSA persistence and very rapid disease trajectories, characterised by early progression to overt metastatic disease and death. Patient 11196_3 had a moderately sized tumour, with only ISUP grade group 3 disease at prostatectomy, but was radiorecurrent, with extensive extracapsular extension and invasion into the seminal vesicle. Prominent lymphovascular invasion, a pathological feature associated with aggressive disease [35], was also observed in large extraprostatic vessels. The second patient 11201_4 had more conventional high-risk disease, with a large volume, locally advanced ISUP grade group 5 tumour. In both cases, pre-treatment staging with conventional imaging was negative for metastatic disease, although the likelihood of tumour cell dissemination at the time of diagnosis was high. Similarly, the cohort we used for the TAm-Seq analysis is a higher risk cohort than that used for ultra-low pass whole-genome analysis, based on a higher median PSA and the proportion of patients with ISUP grade group 4/ 5 disease, which is not unexpected given this study group was enriched for recurrent and metastatic events post-prostatectomy. However, there may be some additional technical issues, as despite including probes for copy number variants in the targeted panel for $7 / 8$ patients (including patients 11196_3 and 11201_4), we were unable to positively identify any ctDNA CNAs concordant with tumour whole-genome sequencing data using this particular custom panel. It may be that even in patients with detectable ctDNA in the clinically localised setting, absolute levels are too low to make confident calls with the sensitivity of the assay used in this study, at least not without a considerable investment is assay development and control samples for normalisation. Certainly, it has been suggested that tumour DNA fractions $>35 \%$ are required to accurately detect CNA events [36], particularly deletions, and this far exceeds the levels observed in our study, even with extremely aggressive localised disease.

A key finding in this study is the consistent association between pre-treatment ctDNA detection and clinical outcomes, which may serve as a useful adjunct to select patients for treatment intensification at the time of diagnosis. Creating a custom panel based on sequencing of the primary tumour and identifying individual-specific variants may be the most sensitive way of positively identifying ctDNA in patients, but outside of particularly invested centres, its use as a clinical strategy is limited by the cost and turn-around time. An alternative approach is to target common variants identified in a landscape-type analysis [21]. We were able to measure SVs and SNVs consistently, suggesting that a strategy 


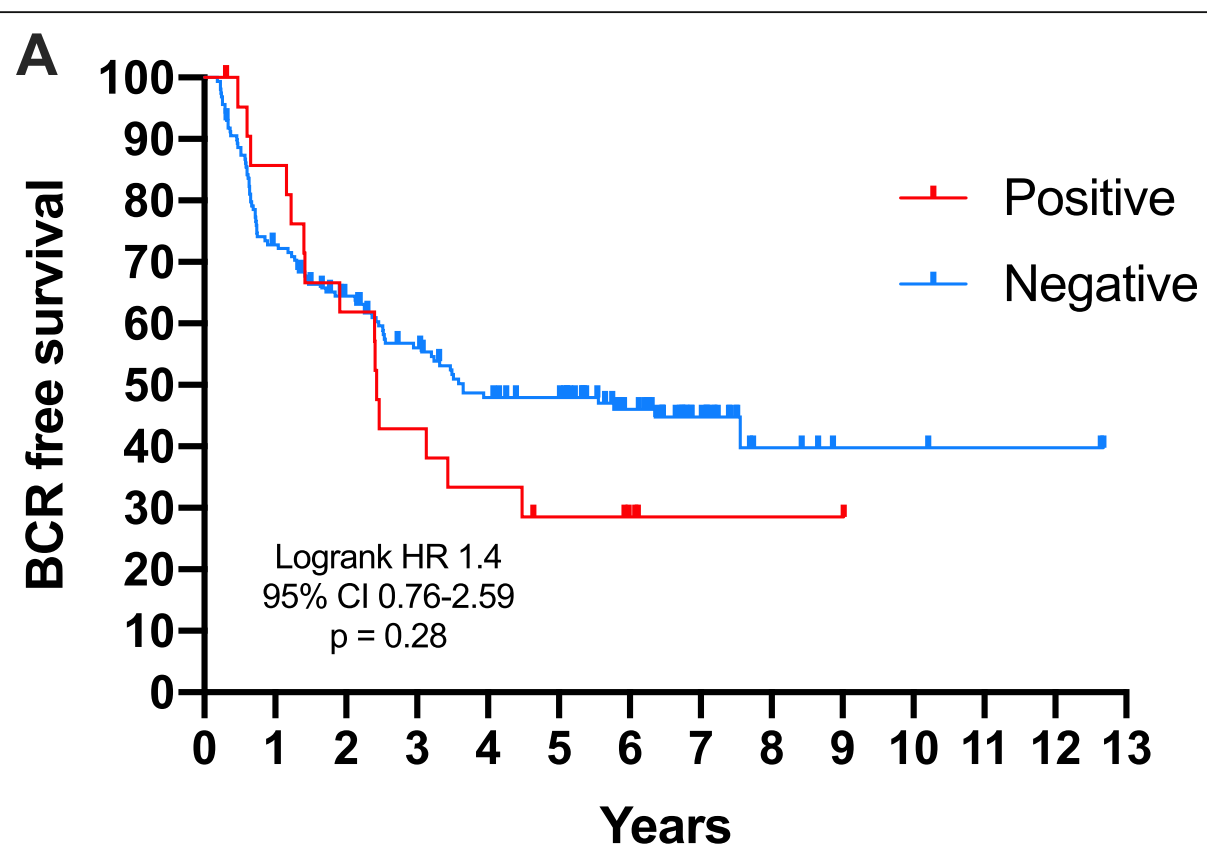

B

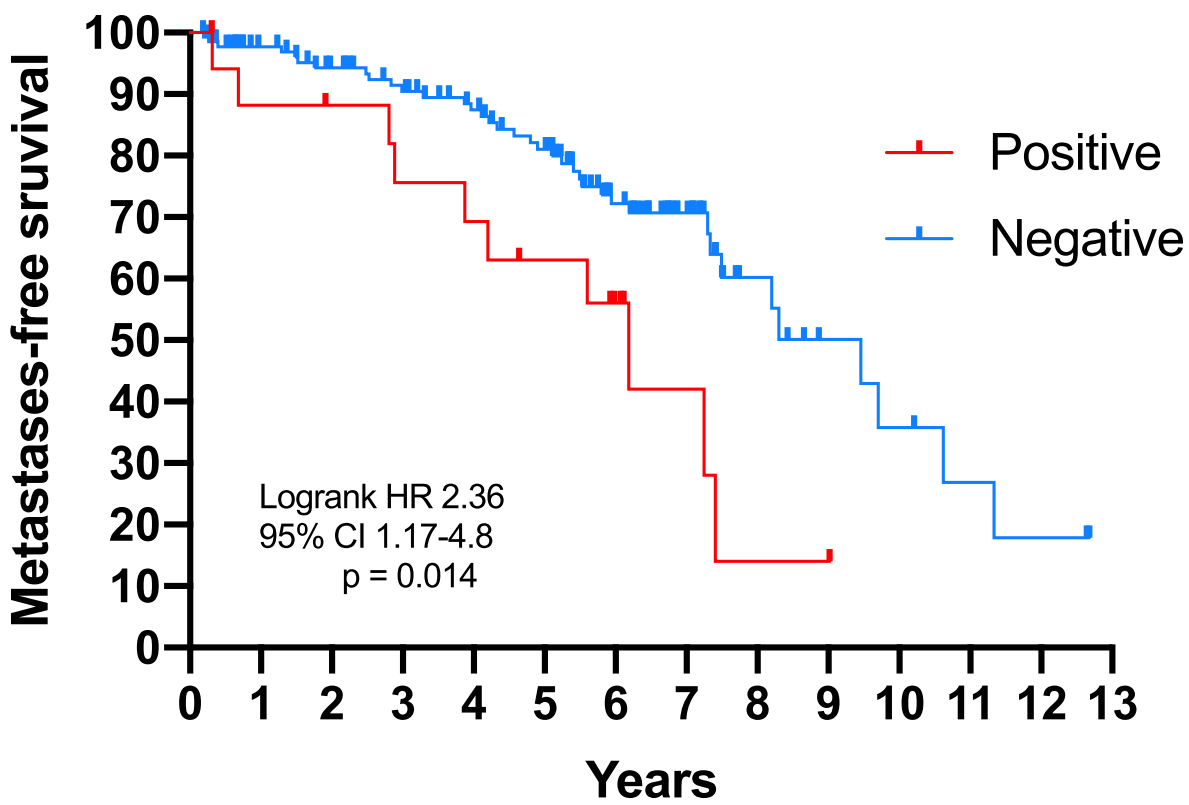

Fig. 4 Kaplan-Meier curves demonstrating a biochemical recurrence and $\mathbf{b}$ metastasis-free survival in a cohort of 189 patients undergoing prostatectomy categorised by pre-treatment TP53 ctDNA status

built around detecting this class of variants based on observed frequency in landscape analysis may be the best approach. One concern however is that SNVs in prostate cancer are rarely located in 'hot-spot' regions, and breakpoint sites vary significantly between patients, requiring coverage of a relatively large genomic region to increase sensitivity. Certainly using this approach tiling across TP53, mutations of which are enriched in patients with metastatic disease, we were able to identify ctDNA in just under $12 \%$ of patients, where it again was associated with the development of metastatic disease. Clearly, all patients with potentially detectable ctDNA levels were not identified, as many patients who were TP53 ctDNA negative also experienced metastatic progression postoperatively. Extending the number of variants assessed will likely increase test sensitivity [37], but for an 'off- 
the-shelf' assay, an optimal 'sweet spot' that balances test complexity, cost and diagnostic performance will need to be determined.

There are a number of limitations to our study that warrant some consideration. Undiagnosed clonal haematopoiesis of indeterminate potential (CHIP), of which TP53 mutation is a relatively frequent driver, has been shown to be a common source of variants detected in deep cell-free DNA analyses and ideally should be controlled for by concomitant sequencing of white blood cell DNA which we have not performed [38, 39]. However, we observe that two of the variants (chr17: 7578475_G>A and chr17:7577121_G>A) identified by TAm-Seq were also detected in previous whole-genome sequencing on the same tumour samples [30]. Enrichment for a known driver of prostate cancer spread in the cohort of patients who progress to clinical metastases suggests that the majority of identified variants are tumour related, but further work is required to confirm this. In addition, the TAm-seq assay had reasonably low sensitivity, which was not unexpected given the reasonably low frequency of TP53 mutations in localised prostate cancer $[20,21]$. However despite these issues with sensitivity and specificity, the TAm-Seq analysis supported the association between ctDNA detection and aggressive disease in the localised setting. It is also important to note that all patients in our study were staged using conventional imaging. Given the reported greater sensitivity of molecular staging, for example, PSMA-PET in detecting low volume disease in the recurrence setting [40], such imaging modalities may better detect patients with micrometastatic disease pretreatment. Whether such imaging gives potentially the same information or is complementary with ctDNA detection will need to be determined.

\section{Conclusions}

In summary, using two separate cohorts and two different approaches, we have found that ctDNA can be identified in patients with clinically localised prostate cancer and is associated with a significantly shorter time to metastatic progression. Efforts to improve levels of detection, specificity and universal applicability of ctDNA detection in this setting are warranted.

\section{Supplementary information}

Supplementary information accompanies this paper at https://doi.org/10. 1186/s13073-020-00770-1.

Additional file 1: Supplementary Tables S1, S3-5. DNA samples analysed and variants identified by WGS; TP53 variants identified by TAmSeq and distribution of variants by clinical outcome.

Additional file 2: Supplementary Table S2. Genomic variants targeted for deep sequencing.
Additional file 3: Supplementary Figure S1-S3. Probe design strategy for panel sequencing and primer/amplicon map for TAm-Seq; clinical outcomes of additional patients.

Additional file 4: Supplementary Methods. Detailed description of TAm-Seq methodology.

\section{Abbreviations}

ADT: Androgen deprivation therapy; BFS: Biochemical recurrence-free survival; Cl: Confidence interval; CNA: Copy number aberration;

mCRPC: Metastatic castration-resistant prostate cancer; CTC: Circulating tumour cell; ctDNA: Circulating tumour DNA; HR: Hazard ratio;

IRB: Institutional Review Board; ISUP: International Society of Urological Pathology; MFS: Metastasis-free survival; MRI: Magnetic resonance imaging; PSA: Prostate-specific antigen; PSMA-PET: Prostate-specific membrane antigen-positron emission tomography; RARP: Robot-assisted radical prostatectomy; SNV: Single nucleotide variant; SV: Structural variant; TAmSeq: Tagged-amplicon deep sequencing; UMI: Unique molecular identifier; WGS: Whole-genome sequencing

\section{Acknowledgements}

We thank all the patients (and their families) for participating in the clinical trial and permitting us to use their specimens for this research.

\section{Authors' contributions}

Overall study design: AJC, DJB, MTR, CMH and NMC; collection and processing of clinical samples: PMcC, FR, KC, MC, MK, KP, HN, MH, ZK, HC, AJC, CMH and NMC; clinical data collection and curation: PMCC, FR, KC, MK, MAF, HC and NMC; statistical and bioinformatics analyses: EL, EMK, SM, DJB and BP; supervised research: DJB, MTR, BP, CMH and NMC; wrote first draft of paper: NMC; reviewed and approved text: all authors.

\section{Funding}

TAm-Seq analysis was funded by a philanthropic grant from Perpetual Trustees, Australia (PI NMC). Support for analysis was provided through the PRECEPT program grant, co-funded by Movember and the Australian Federal Government (PI NMC). BP was supported by a Victorian Health and Medical Research Fellowship from the Department of Health and Human Services in the State of Victoria. NMC was supported by a David Bickart Clinician Researcher Fellowship from the Faculty of Medicine, Dentistry and Health Sciences, University of Melbourne, and more recently by a Movember Distinguished Gentleman's Ride Clinician Scientist Award through the Prostate Cancer Foundation of Australia's Research Program.

\section{Availability of data and materials}

Bam files for WGS, targeted panel sequencing and TAm-Seq are available through the European Genome-phenome Archive, EGA, accession ID EGAS00001004636.

\section{Ethics approval and consent to participate}

All patients provided written informed consent before enrolment. All study interventions and investigations were approved by the Human Research Ethics Committee at Epworth Hospital (HREC 626-14) or Melbourne Health (HREC 2011.009). All research procedures conformed to the principles of the Helsinki Declaration.

Consent for publication

Not applicable.

Competing interests

The authors declare that they have no competing interests.

\section{Author details}

${ }^{1}$ Department of Surgery, University of Melbourne, 5th Floor Clinical Sciences Building, Royal Melbourne Hospital, Grattan Street, Parkville, VIC 3050, Australia. ${ }^{2}$ Melbourne Bioinformatics, The University of Melbourne, Carlton, VIC 3053, Australia. ${ }^{3}$ Department of Medicine, School of Clinical Sciences, Monash University, Melbourne, VIC 3800, Australia. ${ }^{4}$ Department of Medical Oncology, Monash Health, Melbourne, VIC 3168, Australia. ${ }^{5}$ llumina Cambridge Ltd., Great Abington, Cambridge, UK. ${ }^{6}$ Division of Bioinformatics, Walter and Eliza Hall Institute, Parkville, VIC 3052, Australia. ${ }^{7}$ Australian 
Prostate Cancer Centre, North Melbourne, VIC 3195, Australia. ${ }^{8}$ Department of Urology, Royal Melbourne Hospital, Melbourne, VIC 3050, Australia. ${ }^{9}$ Department of Urology, Inselspital, Bern University Hospital, Bern, Switzerland. ${ }^{10}$ Department of Clinical Pathology, The University of Melbourne, Victorian Comprehensive Cancer Centre, Melbourne, VIC 3000, Australia. ${ }^{11}$ Department of Urology, Peninsula Health, Frankston, VIC 3199, Australia. ${ }^{12}$ Victorian Comprehensive Cancer Centre, Melbourne, VIC 3000, Australia.

Received: 19 April 2020 Accepted: 30 July 2020

Published online: 17 August 2020

\section{References}

1. Hamdy FC, Donovan JL, Lane JA, et al. 10-year outcomes after monitoring, surgery, or radiotherapy for localized prostate cancer. N Engl J Med. 2016; 375:1415-24.

2. D'Amico AV, Whittington $R$, Malkowicz SB, et al. Pretreatment nomogram for prostate-specific antigen recurrence after radical prostatectomy or external-beam radiation therapy for clinically localized prostate cancer. J Clin Oncol. 1999;17:168-72

3. Cooperberg MR, Broering JM, Carroll PR. Risk assessment for prostate cancer metastasis and mortality at the time of diagnosis. J Natl Cancer Inst. 2009; 101:878-87.

4. Davis ID, Martin AJ, Stockler MR, et al. Enzalutamide with standard first-line therapy in metastatic prostate Cancer. N Engl J Med. 2019;381:121-31.

5. Armstrong AJ, Szmulewitz RZ, Petrylak DP, et al: ARCHES: a randomized, phase III study of androgen deprivation therapy with enzalutamide or placebo in men with metastatic hormone-sensitive prostate cancer I Clin Oncol 37:2974-2986, 2019.

6. Chi KN, Agarwal N, Bjartell A, et al. Apalutamide for metastatic, castrationsensitive prostate cancer. N Engl J Med. 2019;381:13-24.

7. James ND, de Bono JS, Spears MR, et al. Abiraterone for prostate cancer not previously treated with hormone therapy. N Engl J Med. 2017;377:338-51.

8. James ND, Sydes MR, Clarke NW, et al. Addition of docetaxel, zoledronic acid, or both to first-line long-term hormone therapy in prostate cancer (STAMPEDE): survival results from an adaptive, multiarm, multistage, platform randomised controlled trial. Lancet. 2016;387:1163-77.

9. Fizazi K, Tran N, Fein L, et al. Abiraterone plus prednisone in metastatic, Castration-Sensitive Prostate Cancer. N Engl J Med. 2017;377:352-60.

10. Sweeney CJ, Chen YH, Carducci M, et al. Chemohormonal therapy in metastatic hormone-sensitive prostate cancer. N Engl J Med. 2015;373:737-46.

11. Efstathiou E, Davis JW, Pisters L, et al. Clinical and biological characterisation of localised high-risk prostate cancer: results of a randomised preoperative study of a luteinising hormone-releasing hormone agonist with or without abiraterone acetate plus prednisone. Eur Urol. 2019;76:418-24.

12. McKay RR, Ye H, Xie W, et al. Evaluation of intense androgen deprivation before prostatectomy: a randomized phase II trial of enzalutamide and leuprolide with or without abiraterone. J Clin Oncol. 2019:37:923-31.

13. Spratt $D E$, Yousefi $K$, Deheshi $S$, et al. Individual patient-level meta-analysis of the performance of the decipher genomic classifier in high-risk men after prostatectomy to predict development of metastatic disease. J Clin Oncol. 2017;35:1991-8.

14. Klein EA, Cooperberg MR, Magi-Galluzzi C, et al. A 17-gene assay to predict prostate cancer aggressiveness in the context of Gleason grade heterogeneity, tumor multifocality, and biopsy undersampling. Eur Urol. 2014;66:550-60.

15. Freedland SJ, Gerber L, Reid J, et al. Prognostic utility of cell cycle progression score in men with prostate cancer after primary external beam radiation therapy. Int J Radiat Oncol Biol Phys. 2013;86:848-53.

16. Wei L, Wang J, Lampert E, et al. Intratumoral and intertumoral genomic heterogeneity of multifocal localized prostate cancer impacts molecular classifications and genomic prognosticators. Eur Urol. 2017;71:183-92.

17. Corcoran NM, Hong MK, Casey RG, et al. Upgrade in Gleason score between prostate biopsies and pathology following radical prostatectomy significantly impacts upon the risk of biochemical recurrence. BJU Int. 2011; 108:E202-10.

18. Ku SY, Gleave ME, Beltran H. Towards precision oncology in advanced prostate cancer. Nat Rev Urol. 2019;16:645-54.

19. Soekmadji C, Corcoran NM, Oleinikova I, et al. Extracellular vesicles for personalized therapy decision support in advanced metastatic cancers and its potential impact for prostate cancer. Prostate. 2017;77:1416-23.
20. Armenia J, Wankowicz SAM, Liu D, et al. The long tail of oncogenic drivers in prostate cancer. Nat Genet. 2018;50:645-51.

21. Cancer Genome Atlas Research N. The molecular taxonomy of primary prostate cancer. Cell. 2015;163:1011-25.

22. Hennigan ST, Trostel SY, Terrigino NT, et al. Low abundance of circulating tumor DNA in localized prostate cancer. JCO Precis Oncol. 2019;3: PMC6746181. https://doi.org/10.1200/PO.19.00176.

23. Khakoo S, Carter PD, Brown G, et al. MRI tumor regression grade and circulating tumor DNA as complementary tools to assess response and guide therapy adaptation in rectal cancer. Clin Cancer Res. 2020;26:183-92.

24. Christensen E, Birkenkamp-Demtroder K, Sethi H, et al. Early detection of metastatic relapse and monitoring of therapeutic efficacy by ultra-deep sequencing of plasma cell-free DNA in patients with urothelial bladder carcinoma. J Clin Oncol. 2019;37:1547-57.

25. F. Reeves BP, E. Lau, T. Costello, H. Crowe, M. Ross, D. McBride, S. Mangiola, C. Hovens, N. Corcoran: Posters BJU International 125:91, 2020.

26. Kerger $M$, Hong MK, Pedersen J, et al. Microscopic assessment of fresh prostate tumour specimens yields significantly increased rates of correctly annotated samples for downstream analysis. Pathology. 2012;44:204-8.

27. Raczy C, Petrovski R, Saunders CT, et al. Isaac: ultra-fast whole-genome secondary analysis on Illumina sequencing platforms. Bioinformatics. 2013; 29:2041-3.

28. Sondka Z, Bamford S, Cole CG, et al. The COSMIC Cancer Gene Census: describing genetic dysfunction across all human cancers. Nat Rev Cancer. 2018;18:696-705.

29. Kopanos C, Tsiolkas V, Kouris A, et al. VarSome: the human genomic variant search engine. Bioinformatics. 2019;35:1978-80.

30. Hong MK, Macintyre G, Wedge DC, et al. Tracking the origins and drivers of subclonal metastatic expansion in prostate cancer. Nat Commun. 2015;6:6605.

31. Allen D, Butt A, Cahill D, et al. Role of cell-free plasma DNA as a diagnostic marker for prostate cancer. Ann N Y Acad Sci. 2004;1022:76-80.

32. Bastian PJ, Palapattu GS, Yegnasubramanian S, et al. Prognostic value of preoperative serum cell-free circulating DNA in men with prostate cancer undergoing radical prostatectomy. Clin Cancer Res. 2007;13:5361-7.

33. Chun FK, Muller I, Lange I, et al. Circulating tumour-associated plasma DNA represents an independent and informative predictor of prostate cancer. BJU Int. 2006;98:544-8.

34. Xie $W$, Regan MM, Buyse $M$, et al. Metastasis-free survival is a strong surrogate of overall survival in localized prostate cancer. J Clin Oncol. 2017; 35:3097-104.

35. Galiabovitch E, Hovens CM, Peters JS, et al. Routinely reported 'equivocal' lymphovascular invasion in prostatectomy specimens is associated with adverse outcomes. BJU Int. 2017;119:567-72.

36. Sumanasuriya S, Omlin A, Armstrong A, et al. Consensus statement on circulating biomarkers for advanced prostate cancer. Eur Urol Oncol. 2018;1: 151-9.

37. Wan JCM, Heider K, Gale D, et al: High-sensitivity monitoring of ctDNA by patient-specific sequencing panels and integration of variant reads. Sci Transl Med. 2020;12(548):eaaz8084. https://doi.org/10.1126/scitransImed. aaz8084.

38. Xie M, Lu C, Wang J, et al. Age-related mutations associated with clonal hematopoietic expansion and malignancies. Nat Med. 2014;20:1472-8.

39. Razavi P, Li BT, Brown DN, et al. High-intensity sequencing reveals the sources of plasma circulating cell-free DNA variants. Nat Med. 2019;25: 1928-37.

40. Fendler WP, Calais J, Eiber M, et al. Assessment of 68Ga-PSMA-11 PET accuracy in localizing recurrent prostate cancer: a prospective single-arm clinical trial. JAMA Oncol. 2019;5:856-63.

\section{Publisher's Note}

Springer Nature remains neutral with regard to jurisdictional claims in published maps and institutional affiliations. 